\title{
Cerebellar Liponeurocytoma Mimicking Cerebellopontine Angle Epidermoid Cyst: A Case Report
}

\author{
Oumar Coulibaly ${ }^{1 *}$, Seylan Diawara ${ }^{1}$, Nizar Fatemi ${ }^{1}$, Rachid Gana ${ }^{1}$, Amar Saïdi², \\ Rachid Maaqili', Mohamed Jiddane ${ }^{3}$, Fouad Bellakhdar' \\ ${ }^{1}$ Department of Neurosurgery, CHU Ibn Sina, Rabat-Salé, Morocco \\ ${ }^{2}$ Center of Neuropathology, Rabat, Morocco \\ ${ }^{3}$ Department of Neuroradiology, CHU Ibn Sina, Rabat-Salé, Morocco \\ Email: "coulibalynch1@gmail.com
}

Received 10 January 2014; revised 9 February 2014; accepted 8 March 2014

Copyright (C) 2014 by authors and Scientific Research Publishing Inc.

This work is licensed under the Creative Commons Attribution International License (CC BY). http://creativecommons.org/licenses/by/4.0/

c) (i) Open Access

\begin{abstract}
Cerebellar liponeurocytoma (CL) described under many different names, is a rare WHO grade I or II well differentiated neurocytic tumor of the cerebellum with focal lipomatous differentiation. Mainly reported in adulthood, it is fought to be a posterior fossa benign tumor. In this paper, we talk about a 64-year-old woman, following up for Parkinson's disease presented in our department for $\mathbf{7}$ months' history of headache and gait disturbance. MRI showed a right cerebellopontine angle (CPA) heterogeneous unlimited tumor mimicking an epidermoid cyst. She underwent a lateral suboccipital craniectomy procedure that permitted obtaining the whole gross total resection of this tumor. The histopathological diagnosis was a cerebellar liponeurocytoma. She was discharged from hospital the 8th day after surgery and was free of symptoms since 5 years.
\end{abstract}

\section{Keywords}

Cerebellar Liponeurocytoma, Heterogeneous Tumor, Epidermoid Cyst

\section{Introduction}

Cerebellar liponeurocytoma is a rare tumor of the posterior fossa described at the first time by Bechtel et al. in 1978 [1]; and since this date, it has been reported more than 42 times in the literature under many different names including lipomatous medulloblastoma, lipidized medulloblastoma, medullocytoma, and lipomatous

*Corresponding author. 
glioneurocytoma [2]-[6]. Now, this tumor is recognized to be a distinct pathological entity in the revised WHO classification of tumors of the central nervous system, 2000, and considered like a rare WHO grade I or II well differentiated neurocytic tumor of the cerebellum with focal lipomatous differentiation [7]. In this paper, we talk about another case of cerebellar liponeurocytoma with clinical and radiological studies mimicking a huge cerebellopontine angle (CPA) epidermoid cyst in a 64 years old woman.

\section{Case Report}

A 64 years old-woman follow up for Parkinson's disease since 10 years, had been admitted within our department for 07 months history of progressive occipital headache, right hemiparesis and gait disturbance without vomiting and visual loss. Neurological examination only found a right hemiparesis (strength $3 / 5$ ) with increased right superficial reflex and Babinski on right, horizontal nystagmus on the right lateral gaze and right hypoacousia. There was no papilledema in ophthalmological test. MRI showed a right CPA heterogeneous unlimited tumor measuring about $60 \times 40 \times 42 \mathrm{~mm}$ with evident mass effect on the cerebellum, fourth ventricle and the brainstem. Minimal enlargement of the third and lateral ventricles was also seen. On T1 weighted images (T1WI) the tumor was dishomogeneously hypointense with slightly enhancing after gadolinium injection and hyperintense on T2 weighted images. There was minimal perilesional edema (Figures 1(a)-(d)). These characteristics

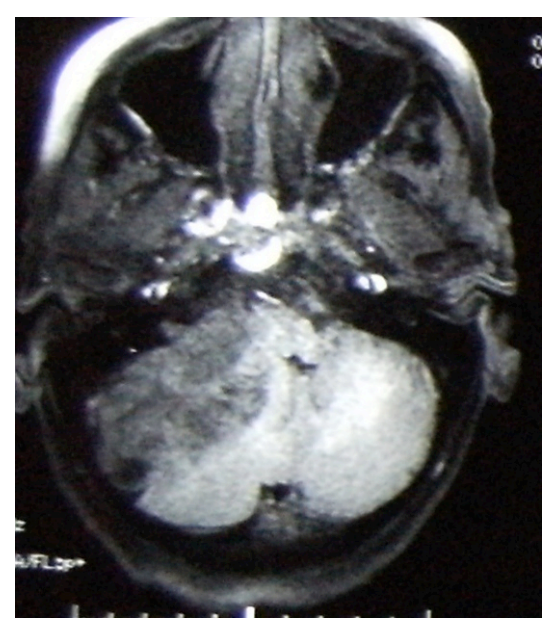

(a)

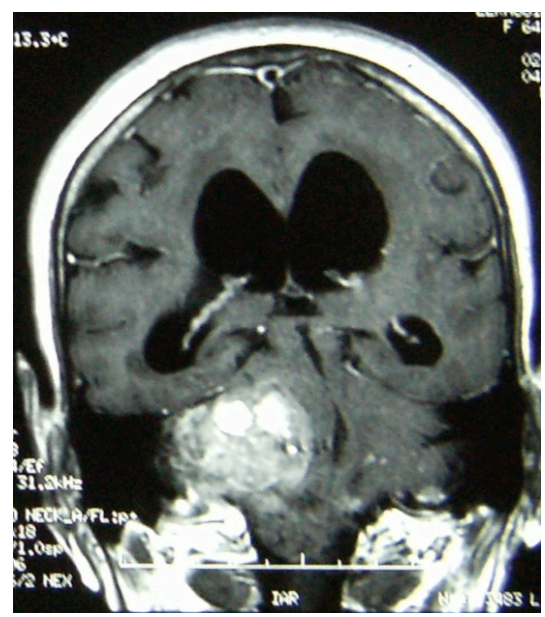

(c)

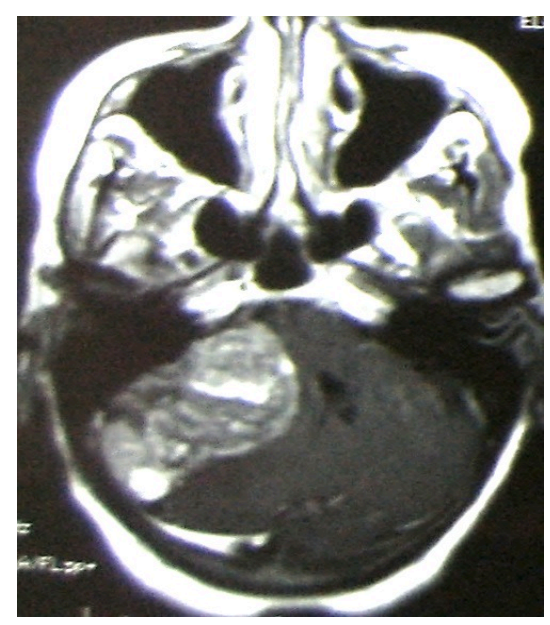

(b)

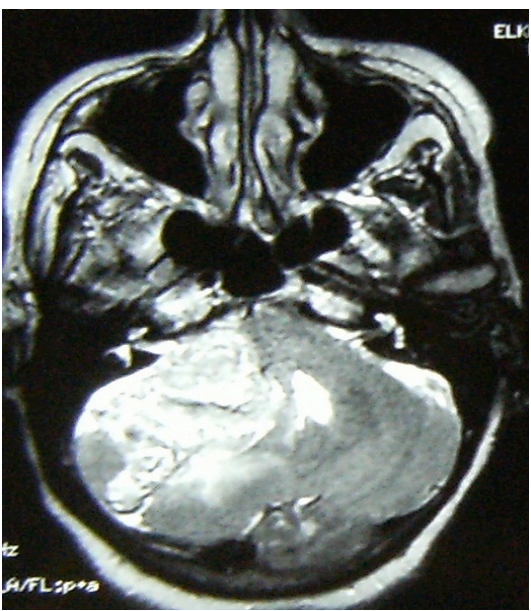

(d)

Figure 1. MRI T1W1 images views showing a heterogeneous CPA mass hypointense to the cerebellum (a) with slightly gadolinium enhancement on axial (b) and coronal (c) views. This lesion is predominantly hyperintense on T2 MRI axial view (d) with evident mass effect on the cerebellum, fourth ventricle and the brainstem. 
were thought to be an epidermoid cyst. She underwent a lateral suboccipital craniectomy procedure that permitted to obtain the wholly gross total resection of this tumor.

Histological examination revealed a neoplasm composed of neurocytes, uniform small cells with round to oval nuclei and scant oligo-like cytoplasm, besides prominent lipidization focicontaining cells resembling adipocytes. Necrosis and microvascular proliferation were absent. Mitoses were scant or absent. The histopathological diagnosis was a cerebellar liponeurocytoma (Figure 2). She was discharged from hospital the $8^{\text {th }}$ day after surgery and is free of symptoms since five years and without adjuvant therapy.

\section{Discussion}

In 2000, the Central Nervous System tumors classification established by the World Health Organisation (WHO) includes a subset called cerebellar liponeurocytoma, a separate clinicopathological entity, corresponding to Grade I or Grade II tumor with a much more favorable prognosis than medulloblastoma [7] [8]. These cerebellar liponeurocytomas are neuroectodermal tumors consisting of both neuronal andglial elements with some interspersed regions of lipidized cells resemble mature adipocytes [6] [9]. Mainly reported in adulthood ranging from 36 to 77 years old (mean age of 53 years), it is fought to be among a posterior fossa benign tumor [3]-[5]. They occur generally in the cerebellum and are characterized by many lipidized cells found in clusters or scattered between small neoplastic cells. Among the 42 cases enrolled in the literature, thirty-six lesions (86\%) were found in the posterior fossa, especially within the cerebellum, and $6(14 \%)$ were found in a supratentorial location [6]. The rare occurrence and diagnosis of this type of tumor as well as its variable appearance on imaging can make radiological diagnosis challenging [10]. On CT it has been reported to be hypo-, iso- or hyperdense. Enhancement with intravenous contrast is variable and often heterogeneous. The lesion may or may not be well demarcated, and can be associated with parenchymal cysts or cerebellar hemorrhage. MRI appearance is also variable and may be related to the distribution and proportion of lipidised tissue. On T1-weighted MRI the mass is generally hyperintense but heterogeneous [11]. Hyperintense "streaks" on T2-weighted images have been associated with the macroscopic appearance of adipose tissue at surgery [2]. Enhancement with gadolinium is usually heterogeneous with areas of tumour demonstrating variable degrees of enhancement. There is either no or minimal associated oedema. The most challenging differential diagnosis of these tumors is to distinguish them from medulloblastomas, ependymomas, solid hemangioblastomas, astrocytomas and metastases [10] [11]. In our case, the tumor was located in the cerebellopontine angle and had also the same characteristics appearances like cerebellopontine angle epidermoid cyst on MRI. This is why this lesion was taken for cerebellopontine angle epidermoid cyst.

This tumor was very well demarcated from the adjacent structures and surgical resection was done success-

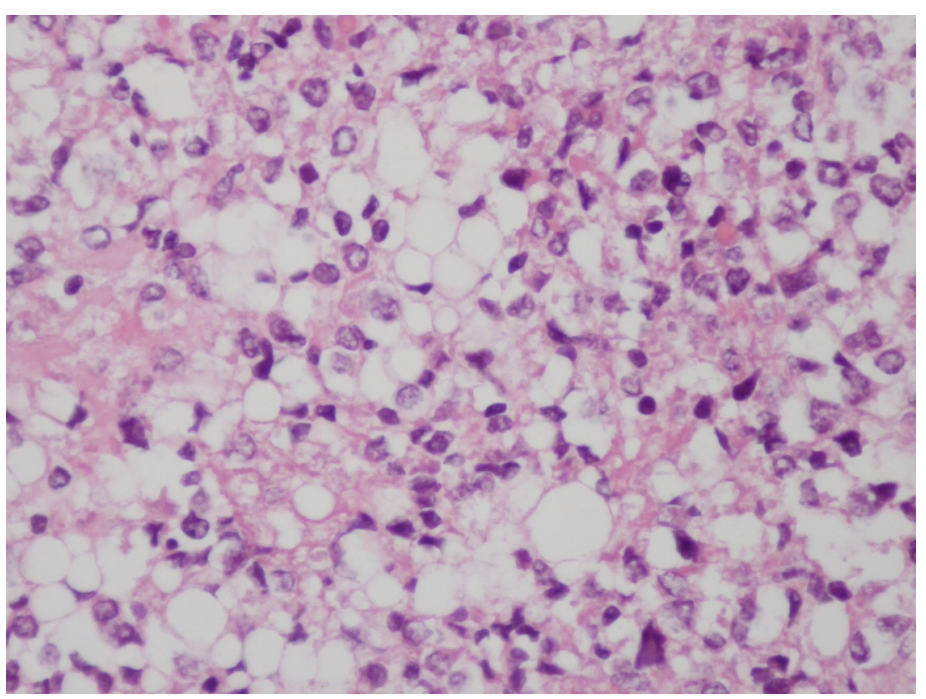

Figure 2. Cytological aspects of cerebellar liponeurocytoma component with scanty cytoplasm and oval or round nuclei with prominent lipid accumulation (HE; ×400). 
fully and permits to have the gross total removal of this mass. But, after histopathological examination, the presence of scanty cytoplasm and oval or round nuclei with prominent lipid accumulation concludes to the diagnosis of liponeurocytoma.

In the literature, surgical resection is recommended as the initial treatment modality for patients with liponeurocytoma to establish a diagnosis. The aim of this surgery is the gross total resection of the tumor. In most of cases there is a reasonable border between the tumor and surrounding tissue and gross total removal of the tumor is possible [10] [12] [13].

Microscopically, liponeurocytoma has small, round toovoid cells with scanty eosinophilic cytoplasm. Lipidized cells resemble mature adipocytes and are found interspersed throughout the lesion (15). Mitoses, areas of vascular proliferation and necrosis are all rare [7] [13] [14].

There is no consensus regarding the treatment of liponeurocytoma, specifically whether chemo- or radiotherapy is a necessary part of the postoperative treatment regimen. In our case, after the gross total resection of this tumor, and through the literature review, we recommend that longer follow-up periods more than 10 years are required to detect some recurrences [6] [15]. Our patient had no additional treatment after total gross removal of this benign tumor and is free of symptoms since five years.

\section{Conclusion}

CPA liponeurocytoma is a rare posterior fossa tumor described since 1978 and mostly seen in adulthood. Radical resection is the treatment of choice to eradicate this kind of lesion, offering such its benignancy regarding this localization.

\section{Acknowledgements}

We would like to thank Mrs. Barry Najah for her supports for this manuscript.

\section{References}

[1] Bechtel, J.T., Patton, J.M. and Takei, Y. (1978) Mixed Mesenchymal and Neuroectodermal Tumor of the Cerebellum. Acta Neuropathologica, 41, 261-263. http://dx.doi.org/10.1007/BF00690447

[2] Alleyne Jr., C.H., Hunter, S., Olson, J.J. and Barrow, D.L. (1998) Lipomatous Glioneurocytoma of the Posterior Fossa with Divergent Differentiation: Case Report. Neurosurgery, 42, 639-643. http://dx.doi.org/10.1097/00006123-199803000-00035

[3] Budka, H. and Chimelli, L. (1994) Lipomatous Medulloblastoma in Adults: A New Tumor Type with Possible Favorable Prognosis. Human Pathology, 25, 730-731. http://dx.doi.org/10.1016/0046-8177(94)90309-3

[4] Davis, D.G., Wilson, D., Schmitz, M. and Markesbery, W.R. (1993) Lipidized Medulloblastoma in Adults. Human Pathology, 24, 990-994. http://dx.doi.org/10.1016/0046-8177(93)90113-U

[5] Giangaspero, F., Cenacchi, G., Roncarolli, F., Rigobello, L., Manvetto, V., Gambacorta, M. and Allegranza, A. (1996) Medullocytoma (Lipidized Medulloblastoma). A Cerebellar Neoplasm of Adults with Favourable Prognosis. American Journal of Surgical Pathology, 20, 656-664. http://dx.doi.org/10.1097/00000478-199606000-00002

[6] Patel, N., Fallah, A., Provias, J. and Jha, N.K. (2009) Cerebellar Liponeurocytoma. Canadian Journal of Surgery, 52, E117-E119.

[7] Kleihues, P. and Cavenee, W.K., Eds. (2000) Pathology and Genetics of Tumours of the Nervous System. World Health Organization Classification of Tumours, Vol. 1, International Agency for Research on Cancer, Lyon.

[8] Jackson, T.R., Regine, W.F., Wilson, D. and Davis, D.G. (2001) Cerebellar Liponeurocytoma. Case Report and Review of the Literature. Journal of Neurosurgery, 95, 700-703. http://dx.doi.org/10.3171/jns.2001.95.4.0700

[9] Owler, B.K., Makeham, J.M., Shingde, M., et al. (2005) Cerebellar Liponeurocytoma. Journal of Clinical Neuroscience, 12, 326-329. http://dx.doi.org/10.1016/j.jocn.2004.05.017

[10] Alkadhi, H., Keller, M., Brander, S., Yonekawa, Y. and Kollias, S. (2001) Neuroimaging of Cerebellar Liponeurocytoma. Journal of Neurosurgery, 95, 324-331. http://dx.doi.org/10.3171/jns.2001.95.2.0324

[11] Akhaddar, A., Zrara, I., Gazzaz, M., El Moustarchid, B., Benomar, S. and Boucetta, M. (2003) Cerebellar Liponeurocytoma (Lipomatous Medulloblastoma). Journal of Neuroradiology, 30, 121-126.

[12] Cacciola, F., Conti, R., Taddei, G.L., Buccoliero, A.M. and Di Lorenzo, N. (2002) Cerebellar Liponeurocytoma: Case Report with Considerations on Prognosis and Management. Acta Neurochirgica (Wien), 144, 829-833.

http://dx.doi.org/10.1007/s007010200082 
[13] Chimelli, L., Hahn, M.D. and Budka, H. (1991) Lipomatous Differentiation in a Medulloblastoma. Acta Neuropathologica, 81, 471-472. http://dx.doi.org/10.1007/BF00293470

[14] Bayar, M., Akif, Pulat, H., Celik, H., et al. (2006) Cerebellar Liponeurocytoma : A Case Report. Turkish Neurosurgery, 16, 150-153.

[15] Soylemezoglu, F., Soffer, D., Onol, B., et al. (1996) Lipomatous Medulloblastoma in Adults. A Distinct Clinicopathological Entity. American Journal of Surgical Pathology, 20, 413-418.

http://dx.doi.org/10.1097/00000478-199604000-00003 